\title{
Perceptual Learning Reduces Crowding in Amblyopia and in the Normal Periphery
}

\author{
Zahra Hussain, Ben S. Webb, Andrew T. Astle, and Paul V. McGraw \\ School of Psychology, University of Nottingham, Nottingham, NG7 2RD, United Kingdom
}

Amblyopia is a developmental visual disorder of cortical origin, characterized by crowding and poor acuity in central vision of the affected eye. Crowding refers to the adverse effects of surrounding items on object identification, common only in normal peripheral but not central vision. We trained a group of adult human amblyopes on a crowded letter identification task to assess whether the crowding problem can be ameliorated. Letter size was fixed well above the acuity limit, and letter spacing was varied to obtain spacing thresholds for central target identification. Normally sighted observers practiced the same task in their lower peripheral visual field. Independent measures of acuity were taken in flanked and unflanked conditions before and after training to measure crowding ratios at three fixed letter separations. Practice improved the letter spacing thresholds of both groups on the training task, and crowding ratios were reduced after posttest. The reductions in crowding in amblyopes were associated with improvements in standard measures of visual acuity. Thus, perceptual learning reduced the deleterious effects of crowding in amblyopia and in the normal periphery. The results support the effectiveness of plasticity-based approaches for improving vision in adult amblyopes and suggest experience-dependent effects on the cortical substrates of crowding.

\section{Introduction}

Amblyopia is a common developmental disorder of spatial vision, characterized by poor visual acuity in one eye with no overt ocular pathology. Two factors contribute to reduced visual acuity in the amblyopic eye: impaired spatial resolution and visual crowding (Flom et al., 1963; Levi and Klein, 1985; Bonneh et al., 2004; Hariharan et al., 2005; Levi et al., 2007). Resolution limits identification of isolated items, whereas crowding arises from the disruptive effects of surrounding stimuli. Standard clinical measures of visual acuity, such as the Bailey-Lovie, Early Treatment Diabetic Retinopathy Study (ETDRS), and Snellen charts, require observers to identify a series of flanked letters at successively smaller angular sizes. It is well established that amblyopic observers show a greater deficit in central (foveal) vision for flanked rather than isolated letters, although such flanking effects are negligible in normal foveal vision (Hariharan et al., 2005). In other words, amblyopes experience crowding at the fovea, in addition to the resolution deficit associated with unflanked items. Crowding effects are ubiquitous in the normal peripheral visual field, and, for this reason, the periphery has often been used as a model (albeit an imprecise one) of the amblyopic fovea (Levi et al., 2007; Levi, 2008). Figure 1 shows crowding measured with

\footnotetext{
Received July 27, 2011; revised Aug. 26, 2011; accepted Nov. 3, 2011.

Author contributions: Z.H., B.S.W., and P.V.M. designed research; Z.H. and A.T.A. performed research; Z.H. analyzed data; Z.H., B.S.W., and P.V.M. wrote the paper.

The authors declare no conflict of interest.

This research has been funded by the European Community's Seventh Framework Programme (FP2007-2013) under Grant Agreement 223326. B.S.W. is funded by a Wellcome Trust Research Career Development Fellowship.

Correspondence should be addressed to Zahra Hussain, School of Psychology, University of Nottingham, Nottingham, NG7 2RD, UK. E-mail: zahra.hussain@nottingham.ac.uk.

DOI:10.1523/JNEUROSCI.3845-11.2012

Copyright $\odot 2012$ the authors $\quad 0270-6474 / 12 / 320474-07 \$ 15.00 / 0$
}

unflanked (isolated) and flanked letters (Fig. $1 A$ ) in the amblyopic fovea (Fig. $1 B$ ) and in the normal lower peripheral visual field (Fig. 1C). For both the amblyopic fovea and normal peripheral field, flanked acuity is substantially worse than unflanked acuity, unlike the negligible effects of flankers on acuity in the normal fovea (Hariharan et al., 2005).

It is known that practice can elicit improvements in several aspects of amblyopic visual function (Levi and Klein, 1985; Levi et al., 1997; Polat et al., 2004; Zhou et al., 2006; Li et al., 2007; Chen et al., 2008; Chung et al., 2008; Huang et al., 2008; Astle et al., 2010; for review, see Levi and Li, 2009). Such learning is relatively permanent and is considered to be the perceptual manifestation of cortical visual plasticity. Although the mechanisms that drive experience-dependent plasticity are particularly malleable early in sensory development, it has become increasingly clear that they can also be activated later in the lifespan. In amblyopic adults, improvements on tasks such as contrast discrimination have been linked with improvements on standard clinical measures of visual acuity (Polat et al., 2004; Chen et al., 2008). However, it is not clear whether the improvements in acuity after perceptual learning are attributable to improvements in resolution or a reduction in crowding or both. Furthermore, it is unknown whether the amblyopic visual system can benefit from perceptual learning directed at the crowding problem per se. Here, we trained amblyopic observers for 8-10 days on a crowded letter identification task to assess whether practice reduces crowding and improves visual acuity. For comparison, we also trained normal observers on the same task in the lower peripheral visual field. Importantly, we measured acuity in both flanked and unflanked conditions before and after training, thereby isolating the relative contributions of resolution and crowding to improvements in visual acuity. 
A
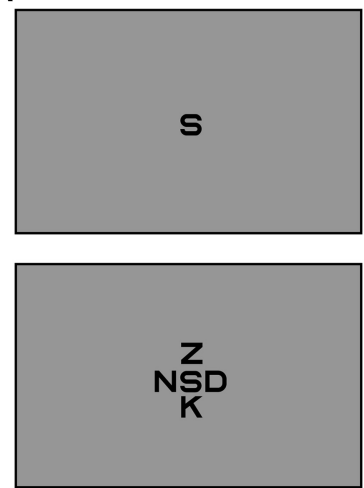

$\mathrm{B}$

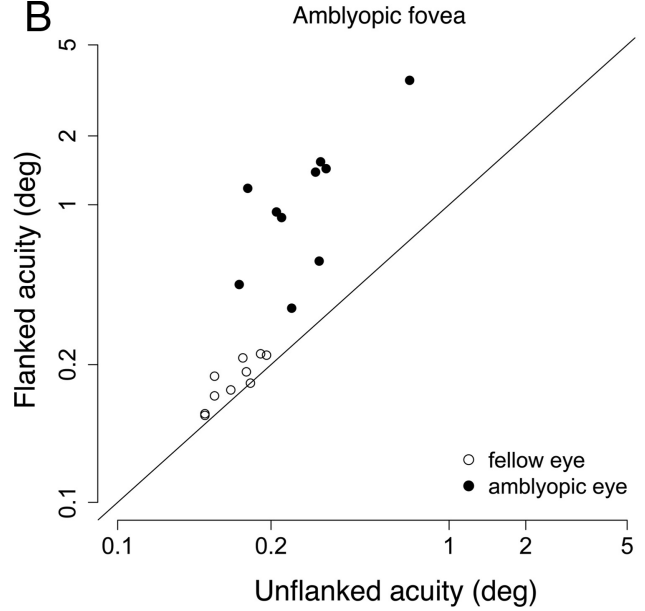

C

C Normal periphery

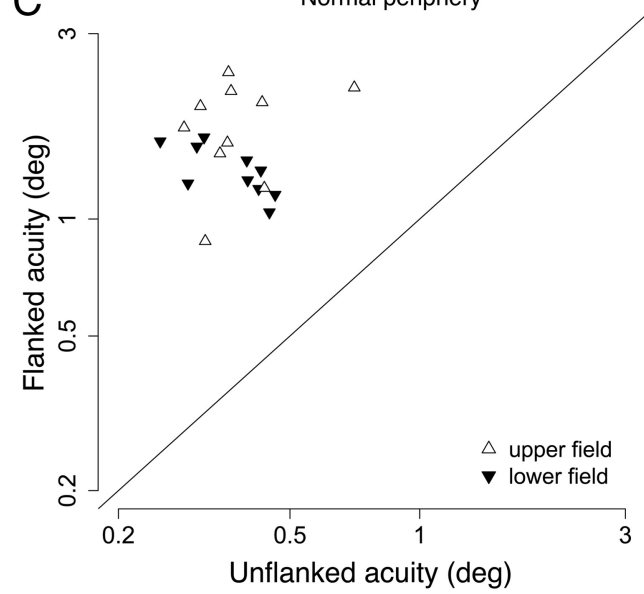

Figure 1. A, Stimuli used in the letter identification task. Unflanked letters (top) and flanked letters (bottom). $\boldsymbol{B}$, Crowding in the amblyopic fovea: acuity thresholds for flanked letters at the closest separation $\left(1.1 \times\right.$ letter size) against acuity thresholds for unflanked letters. Ten subjects were tested. C, Crowding in the normal periphery ( $4^{\circ}$ upper and lower). Ten subjects were tested.

Table 1. Observer clinical details

\begin{tabular}{|c|c|c|c|c|c|c|c|c|}
\hline Participants & Age (years) & Sex & Amblyopic eye & Type & Refractive error & Alignment & logMAR (Pre) & logMAR (Post) \\
\hline S.E. & 17 & M & R & Strab & $\begin{array}{l}0 D+4.50 /-0.75 \times 105 \\
0 S+3.00 /-0.75 \times 95\end{array}$ & RSOT & 0.48 & 0.40 \\
\hline J.A.C. & 45 & M & L & Strab & $\begin{array}{l}0 D+0.25 /-1.00 \times 175 \\
0 S+0.75 /-0.25 \times 50\end{array}$ & LSOT & 0.62 & 0.50 \\
\hline A.P. & 45 & M & L & Strab & $\begin{array}{l}\text { OD }-0.50 /-0.75 \times 175 \\
\text { OS plano/- } 0.50 \times 175\end{array}$ & LSOT & 0.96 & 0.66 \\
\hline J.A. & 21 & $F$ & L & Strab & $\begin{array}{l}O D+3.50 /-0.50 \times 145 \\
O S+4.50 /-0.75 \times 45\end{array}$ & LSOT & 0.54 & 0.42 \\
\hline B.R.C. & 32 & M & R & Strab & $\begin{array}{l}0 \mathrm{D}+3.25 /-2.00 \times 165 \\
\mathrm{OS}+3.75 /-2.25 \times 180\end{array}$ & RSOT & 0.34 & 0.30 \\
\hline L.S.G. & 48 & $\mathrm{~F}$ & L & Mixed & $\begin{array}{l}0 D+0.75 /-0.50 \times 15 \\
0 S+1.75 /-2.00 \times 150\end{array}$ & LSOT & 1.12 & 0.94 \\
\hline S.M. & 34 & M & R & Strab & $\begin{array}{l}O D-0.50 \mathrm{DS} \\
\mathrm{OS}-0.50 /-0.50 \times 160\end{array}$ & RSOT & 1.02 & 0.84 \\
\hline C.J.B. & 57 & M & L & Mixed & $\begin{array}{l}\text { OD }+3.00 /-0.25 \times 75 \\
\text { OS }+6.00 \mathrm{DS}\end{array}$ & LSOT & 0.82 & 0.62 \\
\hline M.M. & 70 & $\mathrm{~F}$ & R & Mixed & $\begin{array}{l}O D+4.75 /-0.75 \times 175 \\
0 S+2.00 /-0.75 \times 105\end{array}$ & RSOT & 0.32 & 0.26 \\
\hline G.J. & 29 & M & R & Mixed & $\begin{array}{l}0 D+4.75 /-2.50 \times 10 \\
0 S+0.75 /-0.25 \times 10\end{array}$ & RXOT & 1.06 & 0.82 \\
\hline
\end{tabular}

LogMAR acuity is given for the amblyopic eye and was measured using the Bailey-Lovie chart. F, Female; $\mathrm{M}$, male; L, left; R, right; Strab, strabmismic; OD, Oculus dexter (right eye); 0S, oculus sinister (left eye); LSOT, left esotropia; RSOT, right esotropia; RXOT, right exotropia.

\section{Materials and Methods}

Observers

Ten amblyopic observers were tested in the fovea, and 17 normally sighted observers were tested in the lower peripheral visual field. Table 1 gives clinical details for amblyopic observers. Normally sighted observers were undergraduate students and research staff at the University of Nottingham. All observers were informed of the purpose and procedure of the study and gave written consent to participate. Amblyopic observers provided a detailed ophthalmic history and were refracted by a registered optometrist before testing. Ocular movements, ocular alignment for distance and near, and binocular functions were also examined. The logarithm of the minimum angle of resolution (logMAR) acuity was measured using the Bailey-Lovie chart.

\section{Apparatus and stimuli}

The experiment was performed with an Apple G5 iMac computer, running PsychoPy (Peirce, 2007). The monitor was a Trinitron Dell P1130 with a screen width of $40 \mathrm{~cm}$ and resolution of $1280 \times 1024$ pixels. Mean background luminance was $41 \mathrm{cdm}^{-2}$. Viewing distance was $114 \mathrm{~cm}$. The stimuli used throughout were 10 Sloan letters (CDHKNORSVZ). Sloan font letters are equal in height and width, which is useful when scaling letter size. Sloan letters are also equally discriminable and therefore frequently used in clinical measures of acuity (Pelli et al., 1988). Black letters $\left(0.25 \mathrm{cdm}^{2}\right)$ were presented on the mean luminance (gray) background (Fig. 1). The fixation mark was a black dot subtending $0.2^{\circ}$ of visual angle.

\section{General procedure}

On the first day of the experiment, amblyopic observers were refracted, and a full ophthalmic history was obtained. The criterion for inclusion in the study was 0.2 logMAR difference (or greater) in acuity between the amblyopic and fellow eye. For all tasks, observers were fitted with best optical correction, seated in a darkened room, and viewing was stabilized by a chinrest. For normally sighted observers who performed the tasks in the periphery, eye dominance was determined using the Miles test, and the tasks were performed using the nondominant eye with the dominant eye occluded. On the first day of the experiment, six pretraining acuity measures were taken (see below). LogMAR acuity was measured using the Bailey-Lovie chart. Training began on the second day of the experiment and continued for a minimum of eight sessions on consecutive days (except weekends). On the final day of the experiment, all of the measures taken on the first day were repeated, and acuity was remeasured using the Bailey-Lovie chart. Five of the 10 amblyopic observers completed an 
Amblyopic fovea

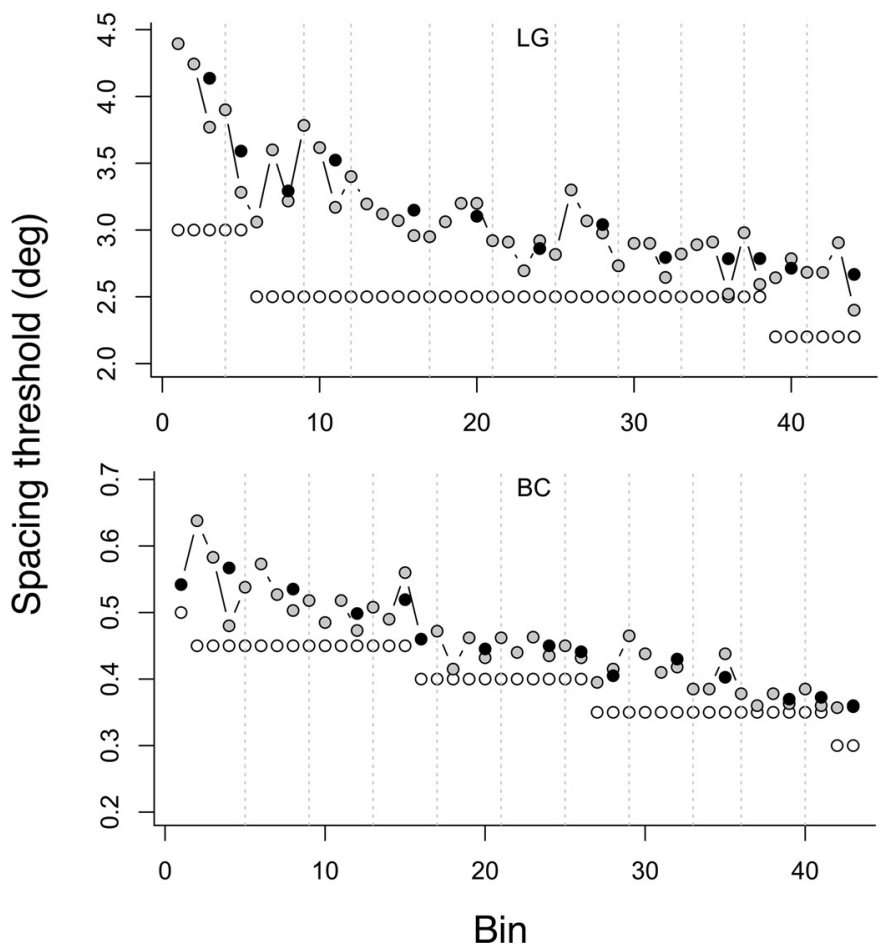

Normal periphery
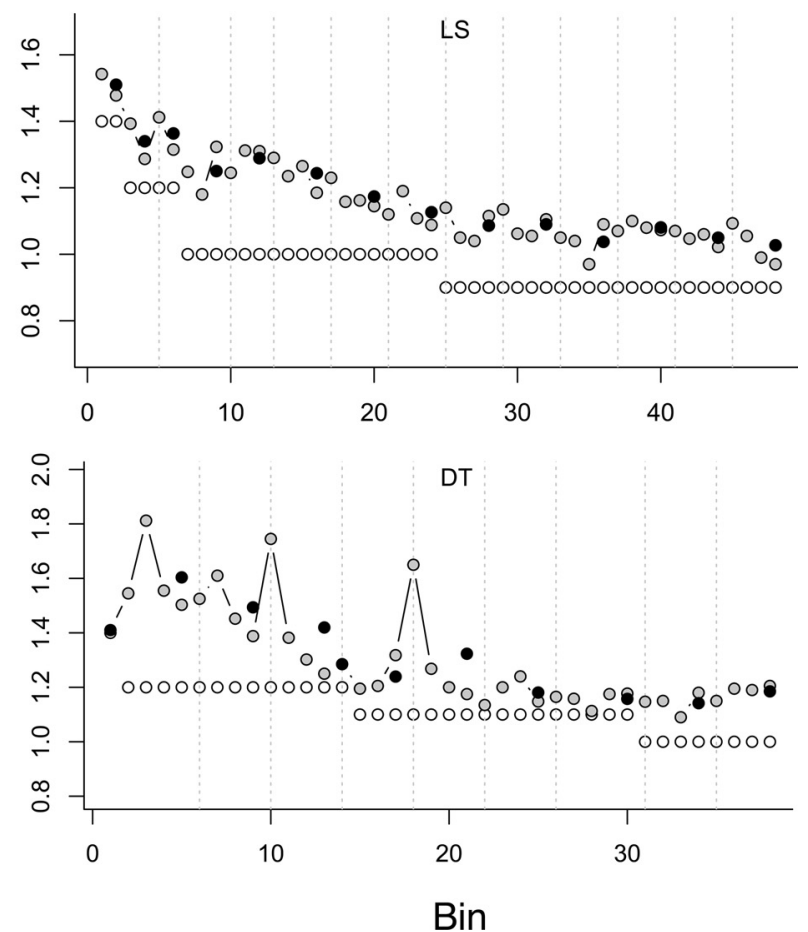

Figure 2. Improvement of two amblyopes (fovea; left) and two normal controls (periphery; right) on the spacing task. Open symbols denote letter size (in degrees) in that bin. Filled gray symbols denote the spacing threshold in that bin. As performance improves, spacing thresholds approach letter size (i.e., abutting). Filled black symbols denote the average spacing threshold for successive bins within a session, at a given letter size. Vertical dashed lines separate the sessions. Sessions were performed on consecutive days.

additional pretest, with no intervening training. These five observers performed the first pretests and returned after 1 week to perform the second pretests. Training began the day after the second pretests were completed. A separate group of seven normally sighted observers performed the pretests and posttests in the periphery without intervening training.

Tasks

Pretraining and posttraining acuity tasks. Letter acuity was measured in flanked and unflanked conditions to compute a crowding ratio at three fixed proportional letter separations $(1.1 \times, 1.2 \times$, and $1.4 \times$ letter size). In the unflanked condition, the target letter was presented alone at fixation in the center of the screen. In the flanked conditions, the target letter was surrounded by four letters, one at each cardinal orientation, with letter separations measured center-to-center, at a fixed proportion of target size $(1.1 \times, 1.2 \times$, and $1.4 \times$ letter size $)$; all conditions were blocked. The observer's task was always to identify the central target letter. In this task, letter size and letter spacing were coupled, such that the absolute spacing between letters scaled with letter size during the task. Each amblyopic observer provided six pretraining and posttraining acuity measures: (1) unflanked acuity in the fellow eye, (2) unflanked acuity in the amblyopic eye, (3) flanked acuity in the fellow eye at a spacing of $1.1 \times$ letter size, and (4) flanked acuities in the amblyopic eye at spacings of $1.1 \times, 1.2 \times$, and $1.4 \times$ letter size. These measures provided an estimate of the crowding ratio in the fellow eye for a letter separation of $1.1 \times$ letter size and crowding ratios in the amblyopic eye for separations of $1.1 \times$, $1.2 \times$, and $1.4 \times$ letter size. Ten normally sighted observers performed the tasks at $4^{\circ}$ in the peripheral field. These 10 observers did eight pretests on day 1 , four each in the upper and lower visual field: (1) unflanked acuity and $(2)$ flanked acuity at the three letter spacings of $1.1 \times, 1.2 \times$, and $1.4 \times$ letter size. The different conditions were blocked, and a minimum of 100 trials was used to obtain threshold in each case, using a three-downone-up staircase.

Practice: letter spacing task. The task was to identify the central target letter in the flanked configuration described above. The size of the target was set according to the size threshold measured at $1.4 \times$ letter size, and letter spacing was varied to establish the minimum separation for $79 \%$ correct performance (three-down-one-up staircase). An initial run of 75 trials was used to ensure that observers could perform the task at the given size. This method provided a reliable estimate of the appropriate difficulty level for each observer. Letter spacing was initially set to twice the letter size and adjusted in linear steps of decreasing magnitude. The minimum spacing in the staircase was equal to letter size (i.e., abutting with center-tocenter spacing). Observers completed three to four blocks of 150 trials per block (450-600 trials per session; 8 sessions minimum for all observers, up to 14 sessions for amblyopes, and 12 sessions for normal observers). Each block yielded a spacing threshold, calculated as the arithmetic mean of the last six reversals. If spacing thresholds approached the minimum value (abutting) for more than two blocks, letter size was decreased by $10 \%$.

For both the above tasks, a trial sequence was as follows: fixation (500 $\mathrm{ms})$, target presentation $(150 \mathrm{~ms})$, blank screen until response. Responses were made by typing in the letter on a keyboard specially designed for visually impaired people. Two of the participants (L.S.G. and M.M.) were unable to respond using the keyboard, so they spoke their response aloud, which was typed into the keyboard by an assistant who was shielded from the display. Auditory feedback was given for all responses (correct/incorrect). The next trial began $1 \mathrm{~s}$ after response.

\section{Results}

We first show how practice gradually improved performance on the letter spacing task in both the amblyopic fovea and normal peripheral visual field. Figure 2 shows learning curves for two amblyopic and two normally sighted observers. Letter spacing thresholds decreased across sessions, gradually approaching abutting (the minimum obtainable threshold), after which letter size was decreased and the task was repeated again at the new letter size. To quantify the amount of improvement on the spac- 

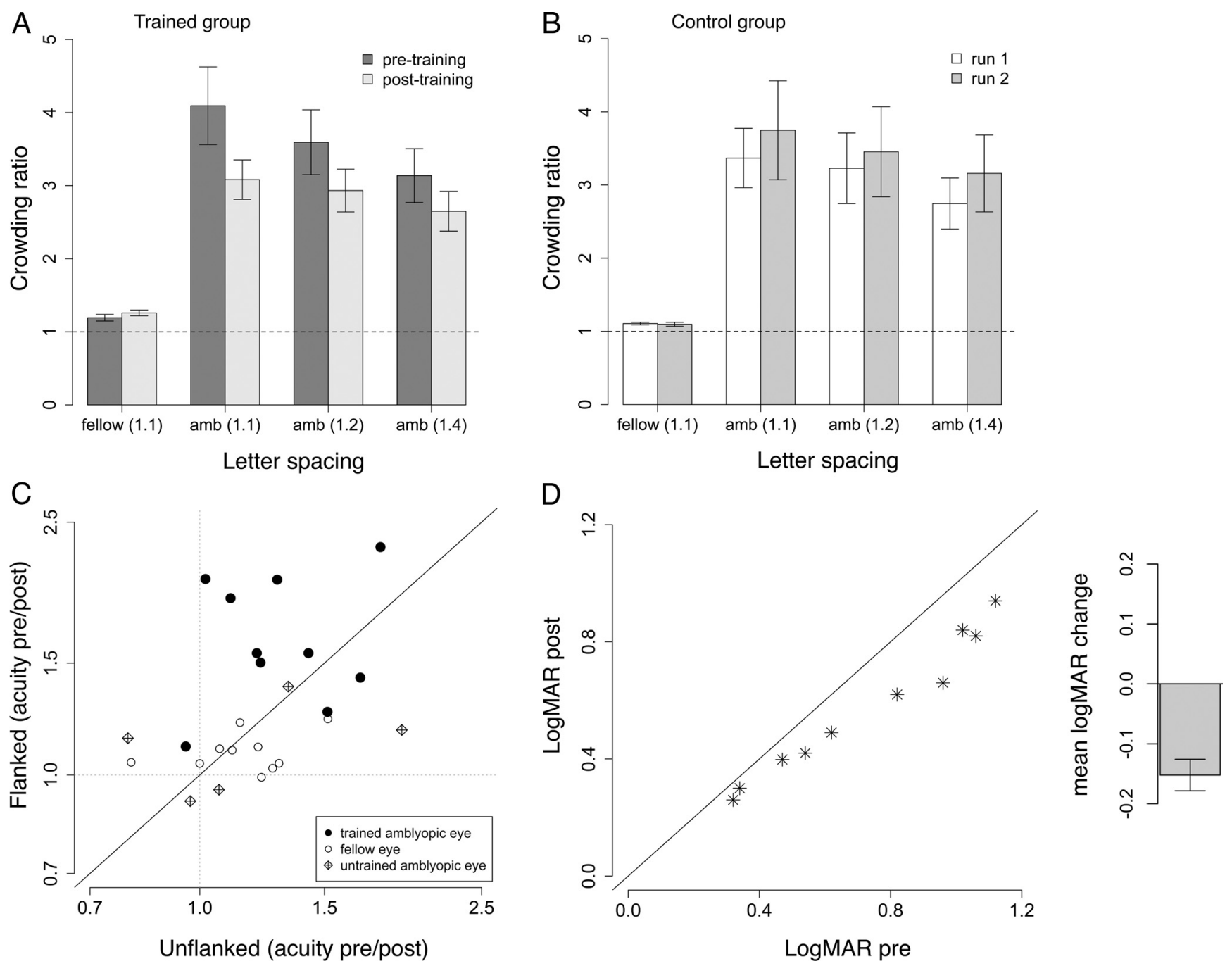

Figure 3. Perceptual learning effects on crowding in the amblyopic fovea. $A$, Pretraining and posttraining crowding ratios (flanked letter acuity/unflanked letter acuity), in trained amblyopes for the amblyopic eye at three separations $(1.1 \times, 1.2 \times, 1.4 \times$ letter size), and the fellow eye at the closest letter separation $(1.1 \times$ letter size). Fellow eye was not trained. B, Crowding ratios of a subset of the amblyopic observers on two separate occasions with no intervening training. Error bars denote SEM. C, Change in unflanked letter acuity versus flanked letter acuity after training (black circles, amblyopic eye; white circles, fellow eye; diamonds, untrained amblyopic eye). Dashed gray lines indicate a ratio of 1 (i.e., no change). D, Change in logMAR acuity after training. Each symbol represents an observer. Average improvement is shown on the right.

ing task, we expressed spacing thresholds as proportion of letter size $(1=$ abutting $)$ and took the difference between the mean of the initial and final thresholds for all trained sizes. The improvement in spacing thresholds was statistically significant (mean $=$ $\left.0.21, t_{(9)}=8.32, p<0.0001\right)$. Initial $\log$ MAR acuity was not correlated with the amount of improvement $\left(r=-0.25, t_{(8)}=\right.$ $0.75, p=0.4711$ ) or with initial threshold elevation in the spacing task $\left(r=-0.07, t_{(8)}=0.7791, p=0.83\right)$, suggesting that crowding is independent of baseline acuity. Improvement in the normal lower periphery was estimated as above, and significant improvement was found (mean $=0.23, t_{(9)}, p=0.00056$ ).

We measured conventional crowding ratios (flanked/unflanked acuity; Fig. $3 A$ ) at three fixed letter separations. Crowding ratios in the normal fovea are $\sim 1$; amblyopic crowding ratios, which were significantly $>1$ in the fovea, decreased after practice (repeated-measures ANOVA; main effect of session, $F_{(1,9)}=$ $7.514, p=0.022)$, Crowding ratios were lower at larger spacings (significant main effect of spacing, $F_{(2,18)}=31.715, p<0.0001$ ), and there was some suggestion that practice differentially affected crowding at the different spacings (marginally significant inter- action between session $\times$ spacing, $\left.F_{(2,18)}=2.89, p=0.0813\right)$. For the control amblyopic observers who did not receive training between sessions (Fig. 3B), crowding ratios did not change (no effect of session, $\left.F_{(1,4)}=0.6483, p=0.4659\right)$.

We examined whether the changes in crowding ratios consisted of improvements in flanked acuity alone or whether unflanked acuity (i.e., resolution) also improved. Figure $3 C$ shows improvements in unflanked and flanked acuities for each amblyopic observer at the closest letter separation $(1.1 \times$ letter size $)$. Unflanked acuity improved for 9 of 10 subjects, and the mean improvement across observers was statistically significant [mean pretest/posttest ratio $(1.32)$ was $\left.>1, t_{(9)}=3.62, p=0.005\right]$. Flanked acuity improved for all 10 observers (mean ratio $=1.66$, $t_{(9)}=5.55, p=0.0003$ ). For 8 of 10 observers, the improvement in flanked acuity was greater than that in unflanked acuity, and the overall mean difference in improvement between flanked and unflanked letters was statistically significant (mean difference $=$ $\left.0.34, t_{(9)}=2.54, p=0.03\right)$. Changes in acuity from the fellow eye of trained amblyopes and untrained amblyopic eyes of five control observers are also shown in Figure 3C. In the fellow eye, 

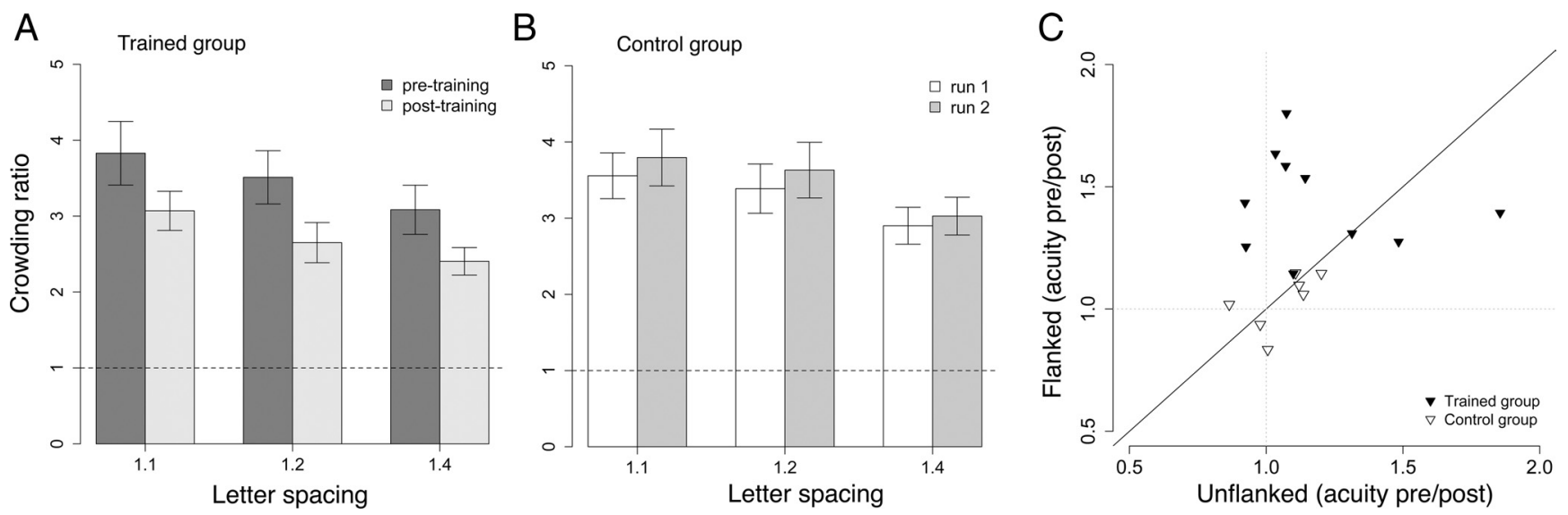

Figure 4. Perceptual learning effects on crowding in the normal periphery. $A$, Pretraining and post-training crowding ratios (flanked letter acuity/unflanked letter acuity), for the trained group at three separations $(1.1 \times, 1.2 \times, 1.4 \times$ letter size). $\boldsymbol{B}$, Pretraining and posttraining crowding ratios for the control group at three separations. $\boldsymbol{C}$, Change in unflanked letter acuity versus flanked letter acuity after training for the trained group (black inverted triangles) and the control group (white inverted triangles). Dashed gray lines indicate a ratio of 1 (i.e., no change). Points above the diagonal indicate greater change in flanked than in unflanked letter acuity. Error bars denote SEM.

unflanked acuity improved significantly (mean ratio $=1.16$, $\left.t_{(9)}=2.6876, p=0.024\right)$, and so did flanked acuity (mean ratio $=$ $\left.1.09, t_{(9)}=3.6496, p=0.005\right)$. However, the difference between flanked and unflanked improvement was not statistically significant (mean ratio difference $=0.07, t_{(9)}=1.32, p=0.22$ ). This pattern suggests general improvement of the fellow eye on the letter identification task, not specific to crowded conditions. For the untrained amblyopic eye, unflanked acuity did not improve between pretests (mean ratio $\left.=1.22, t_{(4)}=1.098, p=0.33\right)$, flanked acuity did improve significantly between pretests (mean ratio $=$ $1.11, t_{(4)}=13.11, p=0.0002$ ), and the difference between flanked and unflanked improvement was not significant (mean ratio difference $\left.=0.10, t_{(4)}=0.59, p=0.59\right)$. Overall, these results suggest that training on a crowded letter identification task reduced the amount of crowding in foveal vision in amblyopia.

Figure $3 D$ shows $\log$ MAR acuity (measured on a Bailey-Lovie chart) for the 10 trained amblyopes before and after training. All amblyopes improved on this measure. The average improvement of the group was statistically significant (mean pretest/posttest $\log$ MAR ratio $\left.=1.25, t_{(9)}=9.01, p<0.0001\right)$. These results suggest that letter spacing training produced improvements in traditional clinical measures of acuity.

We found comparable results for the groups of normally sighted observers who were tested at 4 degrees in the lower periphery. Figure 4 shows crowding ratios for the trained (Fig. $4 A$ ) and control group (Fig. $4 B$ ) and their respective change in acuity (Fig. $4 C$ ). For the trained group, crowding ratios decreased across sessions (repeated-measures ANOVA; significant main effects of session, $F_{(1,9)}=10.581, p<0.01$ ), and there was more crowding at fine letter separations (significant main effect of spacing, $\left.F_{(2,18)}=20.801, p<0.0001\right)$. No improvement across sessions was found for the untrained control group (no effect of session, $F_{(1,6)}=0.17, p=0.6929$ ). Figure $4 C$ shows the change in unflanked and flanked $(0.1 \times$ letter size) acuity in the normal periphery for the trained and control group. For the trained group, unflanked acuity did not improve (mean ratio $=1.19$, $\left.t_{(9)}=2.1087, p=0.0642\right)$, whereas there was a significant improvement in flanked acuity (mean ratio $=1.43, t_{(9)}=6.84, p<0.0001$ ). For the untrained group, there was no improvement either in unflanked (mean ratio $=1.10, t_{(6)}=1.711, p=0.13$ ) or flanked acuity (mean ratio $=1.04, t_{(9)}=1.04, p=0.33$ ). These results suggest that practice on the crowding task selectively improved flanked acuity for the trained group in the normal peripheral field.
Table 2. Crowding ratios

\begin{tabular}{lllll}
\hline Participant & Pretest & Posttest & Follow-up 1 & Follow-up 2 \\
\hline J.A. & 4.43 & 2.28 & 3.56 & 1.95 \\
S.M. & 4.92 & 3.81 & 4.11 & 3.64 \\
\hline
\end{tabular}

We were able to call back 5 of the 10 amblyopic participants for follow-up measures, ranging from 3 to 14 months after their posttest. Unflanked acuity and flanked acuity were measured to obtain the crowding ratio at the nearest separation $(1.1 \times$ letter size) for the amblyopic and fellow eye. LogMAR acuity was also measured. Reductions in crowding ratios in the amblyopic eye were partially retained (mean \pm SEM percentage retention, $50 \pm 14 \%$ ). Crowding ratios were unchanged in the fellow eye. Improvements in $\operatorname{logMAR}$ acuity in the amblyopic eye were essentially intact (mean \pm SEM percentage retention, $91 \pm 16 \%$ ). Two participants did additional practice on the spacing task and returned for a second follow-up session (Table 2). S.M.'s first follow-up session was 14 months after posttest. He practiced the spacing task for one session (375 trials) and returned the next day for a second follow-up. J.A.'s first follow-up was 3 months after posttest. She practiced the spacing task for an additional 11 sessions ( $\sim 6600$ trials), and her second follow-up was the day after her final practice session. The data in Table 2 from the two participants who performed two follow-up sessions suggest that one practice session was sufficient to recover S.M.'s slippage and that multiple practice sessions further reduced crowding for J.A.

\section{Discussion}

Practice of flanked letter identification reduced visual crowding in both the amblyopic fovea and the normal periphery. Reductions in crowding were evident in performance on the training task and in the posttraining acuity tests. On the training task, spacing thresholds decreased such that flanking letters eventually abutted the target, indicating that with practice, observers could tolerate extremely fine letter spacings. On the acuity tasks, improvements in flanked letter acuity outweighed improvements in unflanked letter acuity, yielding lower posttraining crowding ratios at all letter spacings. Crowding ratios did not decrease for control observers who performed the acuity tasks on two separate occasions without intervening training. For the amblyopic group who trained on letter identification, decreases in the magnitude 


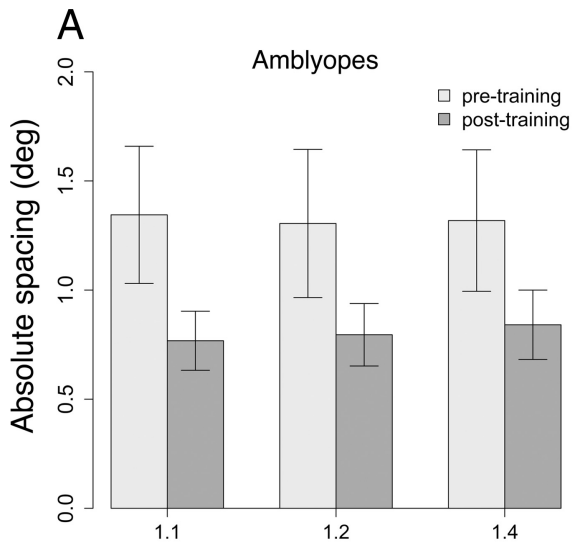

Letter spacing (proportional)
B

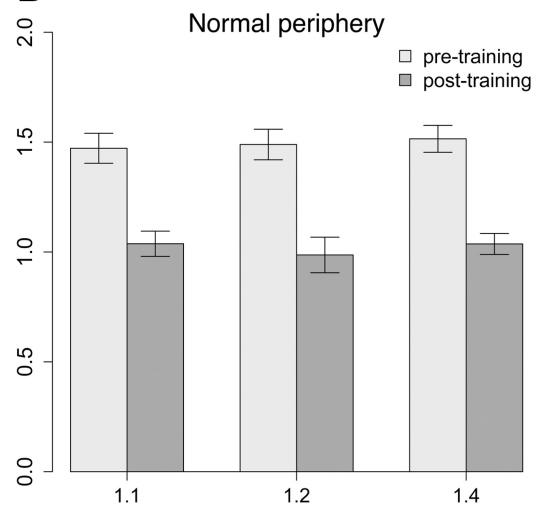

Letter spacing (proportional)

Figure 5. Absolute letter spacings in the flanked acuity tasks. Spacing was a fixed proportion of letter size; therefore, absolute spacings were calculated by multiplying each observer's acuity with the spacing in each condition $(1.1 \times, 1.2 \times, 1.4 \times$ letter size). Error bars denote SEM.

of crowding were accompanied by relatively long-lasting improvements in logMAR acuity, which improved by up to 0.3 $\log$ MAR. Overall, we find that practice of crowded letter identification benefits vision in the amblyopic fovea and is also effective in the peripheral visual field of normal observers.

Practice-induced reductions in crowding have been demonstrated previously in the normal periphery (Chung, 2007; Huckauf and Nazir, 2007; Sun et al., 2010), which is often compared with the amblyopic fovea because of similar losses in acuity and other visual abilities relative to normal central vision. In certain respects, however, the periphery differs from the amblyopic fovea (e.g., receptive field size; Wilson, 1991), and it is not clear that the two share common mechanisms of plasticity (or crowding). It is known that the periphery is more representative of strabismic than anisometropic amblyopia (Levi and Carney, 2011), but there are no direct comparisons of the effects of practice in both cases, or of the effects of "uncrowding" on different aspects of acuity. The present data show equivalent effects of practice on crowding in the normal periphery and in strabismic amblyopes, with additional benefits for unflanked acuity in amblyopes that were not found in the periphery. Interestingly, equivalent amounts of practice reduced crowding by a similar magnitude in both groups, despite greater variation in unflanked acuity within the amblyopic group.

The duration of practice ranged from 8 to 14 sessions, with 450-600 trials per session. This amount of practice significantly reduced but did not abolish crowding either in amblyopes or in the normal periphery. For all observers, residual crowding was still evident in the acuity measures (i.e., crowding ratios were $>1$ ). For amblyopes, it may be that with larger amounts of practice, flanked letter acuity approaches unflanked letter acuity in the fovea. Indeed, when amblyopes' improvements have been reported to approach asymptote on a given task, quite prolonged periods of practice were given (Polat et al., 2004; Li et al., 2007; Chen et al., 2008), and improvements with uncrowded stimuli have been reported to plateau more quickly than with crowded stimuli ( $\mathrm{Li}$ et al., 2007). The method used in the current study afforded observers practice at smaller letter sizes when spacing thresholds approached minimum. Although by the end of training spacing thresholds were near minimum for the given letter size, letter size itself was not at asymptote. Therefore, it is possible that additional practice may have resulted in larger reductions in the magnitude of crowding. The data from one participant who ran for 11 additional sessions after follow-up show further reductions in crowding beyond those measured at posttest (Table 2, J.A.), consistent with the idea that more practice is better and that prolonged practice may reduce crowding to a fraction of initial levels.

It has been suggested that learning in the amblyopic visual system generalizes more widely than in the normal system and that this effect may be dependent on proximity of the trained stimulus to threshold (Huang et al., 2008; Astle et al., 2010). Consistent with this idea, amblyopes' unflanked letter acuity improved after training on the crowded letter spacing task, an effect that was not found in the normal periphery. Furthermore, there were modest improvements in amblyopes' chart acuity after training. We trained all observers under challenging, crowded conditions but at letter sizes that were well above the unflanked letter acuity threshold. Larger letter sizes were chosen to ensure that resolution did not limit performance and to ensure that spacing was the parameter being trained. Training at sizes closer to the (unflanked letter) acuity limit may yield larger reductions in crowding and greater transfer to chart acuity.

We considered the possibility that some proportion of the reduction in crowding may have arisen from nonspecific learning of letter identification in the given conditions (i.e., task-general learning), or from practice on the pre-acuity and post-acuity tests. Indeed, the control data from the fellow eye and the untrained amblyopic eye reflect some improvements in flanked and unflanked acuity at posttest (Fig. 3C). For the fellow eye, significant but equivalent improvements were obtained in flanked and unflanked acuity, leaving crowding ratios unchanged. For the untrained amblyopic eye, improvements in unflanked acuity did not achieve significance, and improvements in flanked acuity did not significantly outweigh unflanked improvements, similar to what was found with the fellow eye. This pattern of results suggests that some task-general learning did occur during the pretests and posttests, but it could not account for the selective reduction in crowding. Crowding was reduced only when improvements in flanked acuity exceeded those in unflanked acuity, after practice on the letter spacing task.

Did the improvements depend on age? Neural plasticity is thought to decline with age, but a recent review of the efficacy of perceptual learning in amblyopia suggests that age is weakly, if at all, correlated with improvements on the trained task or improvements in logMAR acuity (Levi and Li, 2009). We examined correlations between age and a number of outcome measures for the amblyopic participants, who spanned a wide age range in the present study (17-70 years). Age was not significantly correlated with baseline amount of crowding, the amount of improvement on the training task, the reduction in crowding ratio, or improvement in logMAR acuity. Therefore, the benefits of practice on crowding (and logMAR acuity) were not restricted to a particular age group.

Although it appeared that there was less crowding at larger letter spacings in both the amblyopic fovea and the normal periphery, absolute spacing did not vary across conditions (Fig. 5). Because letter spacing was a constant proportion of letter size in the acuity tasks, lower acuity thresholds at larger proportional 
spacings translated as approximately equivalent absolute spacing across the three spacing conditions. The finding that absolute spacing does not vary with acuity (i.e., letter size) is consistent with the idea that a critical spacing rather than a critical size limits performance in crowded conditions (Levi et al., 2002, 2007; Tripathy and Cavanagh, 2002), particularly for strabismic amblyopes (Levi and Carney, 2011).

Crowding is thought to be governed by a two-stage model: the first stage in early visual cortex involves the detection of basic features, and the second integrates these features into an objectbased representation (Pelli et al., 2004; Pelli and Tillman, 2008). Crowding arises when two or more images fall within the same integration zone. If our training regimen had only refined the representation of basic features, we might have expected to see equivalent improvements for flanked and unflanked acuity. The fact that we find additional learning for the flanked case suggests that the feature integration stage is plastic (Chung, 2007) and that the degree of anomalous integration can be ameliorated in adults with amblyopia and in the normal periphery. The flexibility of the spatial extent of crowding also suggests that, at least partly, crowding arises from limited experience with object identification under constraining conditions.

Over the past decade, there has been considerable effort devoted to developing noninvasive treatments for children and adults with amblyopia. Outcome measures of standard clinical treatments do not distinguish between changes in resolution and crowding. We show that each of these aspects of the deficit in amblyopia can be targeted with an appropriate task. Improvements in amblyopic visual acuity have been obtained after practice on contrast detection (Polat et al., 2004; Chen et al., 2008; Huang et al., 2008), vernier acuity (Levi et al., 1997), and position discrimination in noise ( $\mathrm{Li}$ et al., 2007). For reasons that are not clear, improvements in visual acuity were not obtained after training on identification of unflanked letters (Chung et al., 2008). Crowded conditions are not obligatory for improvements in visual acuity (Levi et al., 1997; Chen et al., 2008), but practice with crowded stimuli may yield swifter and more substantial improvements in acuity than those obtained after training with isolated items.

\section{References}

Astle AT, Webb BS, McGraw PV (2010) Spatial frequency discrimination learning in normal and developmentally impaired human vision. Vision Res 50:2445-2454.

Bonneh YS, Sagi D, Polat U (2004) Local and non-local deficits in amblyopia: acuity and spatial interactions. Vision Res 44:3099-3110.

Chen PL, Chen JT, Fu JJ, Chien KH, Lu DW (2008) A pilot study of anisometropic amblyopia improved in adults and children by perceptual learning: an alternative to patching. Ophthalmic Physiol Opt 28:422-428.
Chung ST (2007) Learning to identify crowded letters: does it improve reading speed? Vision Res 47:3150-3159.

Chung ST, Li RW, Levi DM (2008) Learning to identify near-threshold luminance-defined and contrast-defined letters in observers with amblyopia. Vision Res 48:2739-2750.

Flom MC, Weymouth FW, Kahneman D (1963) Visual resolution and contour interaction. J Opt Soc Am 53:1026-1032.

Hariharan S, Levi DM, Klein SA (2005) "Crowding" in normal and amblyopic vision assessed with Gaussian and Gabor C's. Vision Res 45:617-633.

Huang C, Lu Z, Zhou Y (2008) Mechanisms underlying perceptual learning of contrast detection in adults with anisometropic amblyopia. J Vis 9:24.1-24.14

Huckauf A, Nazir TA (2007) How odgcrnwi becomes crowding: stimulusspecific learning reduces crowding. J Vis 7:1-12.

Levi DM (2008) Crowding-an essential bottleneck for object recognition: a mini-review. Vision Res 48:635-654.

Levi DM, Carney T (2011) The effect of flankers on three tasks in central, peripheral and amblyopic vision. J Vis 11:1-23.

Levi DM, Klein SA (1985) Vernier acuity, crowding and amblyopia. Vision Res 25:979-991.

Levi DM, Li RW (2009) Perceptual learning as a potential treatment for amblyopia: a mini-review. Vision Res 49:2535-2549.

Levi DM, Polat U, Hu YS (1997) Improvement in vernier acuity in adults with amblyopia: practice makes better. Invest Ophthalmol Vis Sci 38:1493-1510.

Levi DM, Hariharan S, Klein SA (2002) Suppressive and facilitatory spatial interactions in peripheral vision: peripheral crowding is neither size invariant nor simple contrast masking. J Vis 2:167-177.

Levi DM, Song S, Pelli DG (2007) Amblyopic reading is crowded. J Vis $7: 1-17$.

Li RW, Provost A, Levi DM (2007) Extended perceptual learning results in substantial recovery of positional acuity and visual acuity in juveline amblyopia. Invest Ophthalmol Vis Sci 48:5046-5051.

Peirce JW (2007) PsychoPy: Psychophysics Software in Python. J Neurosci Methods 162:8-13.

Pelli DG, Tillman KA (2008) The uncrowded window of object recognition. Nat Neurosci 11:1129-1135.

Pelli DG, Robson JG, Wilkins AJ (1988) The design of a new letter chart for measuring contrast sensitivity. Clin Vision Sci 2:187-199.

Pelli DG, Palomares M, Majaj NJ (2004) Crowding is unlike ordinary masking: distinguishing feature integration from detection. J Vis 4:1136-1169.

Polat U, Ma-Naim T, Belkin M, Sagi D (2004) Improving vision in adult amblyopia by perceptual learning. Proc Natl Acad Sci US A 101: 6692-6697.

Sun G, Chung ST, Tjan BS (2010) Ideal observer analysis of crowding and the reduction of crowding through learning. J Vis 10:16.

Tripathy SP, Cavanagh P (2002) The extent of crowding in peripheral vision does not scale with target size. Vision Res 42:2357-2369.

Wilson HR (1991) Model of peripheral and amblyopic hyperacuity. Vision Res 31:967-982.

Zhou Y, Huang C, Xu P, Tao L, Qiu Z, Li X, Lu ZL (2006) Perceptual learning improves contrast sensitivity and visual acuity in adults with anisometropic amblyopia. Vision Res 46:739-750. 